\title{
Implementation of ZigBEE (IEEE 802.15.4) Based Wireless ECG Measurement System
}

\begin{tabular}{|c|c|c|}
\hline \multicolumn{3}{|c|}{ Atakan AKBULUT $^{* 1}$, Şükrü EKİN ${ }^{1}$, Ömer TUNÇ, Ali ÖZEN ${ }^{1}$} \\
\hline \multicolumn{3}{|c|}{${ }^{1}$ Nuh Naci Yazgan University-HARGEM, Faculty of Engineering, Electrical and Electronics Engineering, 38010 Kayseri } \\
\hline Başvuru/Received: 08/10/2017 & Kabul/Accepted: 01/12/2017 & Son Versiyon/Final Version: 26/12/2017 \\
\hline
\end{tabular}

\begin{abstract}
This study presents a realization of a ZigBEE based wireless ECG system for remotely monitoring the patient's electrocardiogram (ECG) via wireless heart sensor (AD8232). The presented wireless ECG system is composed of hardware such as Arduino microcontroller connected to the electrodes on the patient, personal laptop, tablet PC, smart phone etc. for ECG signal monitoring, AD8232 sensor for measuring heart rate, ZigBEE transceiver (XBee S2C) for wireless transmission of ECG signal, and software such as Serial Plot or Arduino Serial Plotter. The results from the tests show that the system designed over a single node can work seamlessly in an open area of approximately 20 to 100 meters and take ECG measurements wirelessly. Additionally, the obtained results from the tests show that the ECG measurements can be taken wirelessly within a range of 20 to 80 meters when the obstacles are also placed between ZigBEE transmitter and receiver.
\end{abstract}

Key Words

"Wireless ECG, ZigBEE, Arduino, remote patient monitoring, heart sensor" 


\section{INTRODUCTION}

Important developments have been made in wireless communication systems over the past 50 years. Improvements in highfrequency semiconductor circuits and the reduction of antenna dimensions have contributed significantly to radio communications. Another important factor is the development of battery technology and the efficient use of amplifiers that operate on radio frequency multiples (Somay, 2009).

Establishment of sensor networks with digital radio and the creation of a large-scale network structure is the only way to shape the technology that has been developing and matured for the last few years. This format comes with the emergence of standards such as IEEE 802.15.4 (ZigBEE) (IEEE Standard, 2007).

The integration of biomedical sensors with wireless network technology may have great potentials in medical applications. Technological innovations in the field of disease prevention and patient health protection have led to the development of areas such as monitoring systems. One of the most important developments is the development of real-time monitors that use intelligent and wireless communication technology. The IEEE 802.15.4 standard targets low power, low data rate wireless network applications such as wireless body area network (WBAN), home automation and environmental monitoring network. One of the promising applications of the future is medical care. In advanced hospitals, light and pille-operated sensor nodes equipped with a range of biomedical sensors can be connected to a large number of patients with physiologic data and vital signs. Data such as body temperature, blood pressure, electrocardiogram (ECG), electroencephalography (EEG) and heart rate of patients can be perceived and transmitted to the medical center where these data can be used for monitoring the health status of patients and for further analysis. Biomedical sensor networks can also help ensure freedom of movement while ensuring that patients are constantly monitored and cared (Romer \& Friedemann, 2004).

ECG is a method of examination that allows us to visualize the electrical activity that occurs during the contraction and relaxation phases of the atrium and ventricles of the heart on time and $\mathrm{mV}$ on the millimeter paper. ECG devices are used by the wired method to diagnose cardiac disorders such as fibrillation (shock), heart attack, and cardiac arrhythmia in patients.

Intensive care patients are usually connected to wired ECG devices to provide immediate intervention in any cardiac problems and monitoring with medical devices to reduce deaths. However, the inadequate equipment of ECG devices is a big problem. Researches are being conducted all over the world for the development of these devices. In this system intended to be performed in this study, it is aimed to transmit more than one heart signal wirelessly. In this way, it is aimed to increase the speed of sudden intervention and decrease in cost.

Many procedures are required to obtain the ECG signal from human beings. The working principle of the human body comes in the form of electricity signals and nerve conduction in $\mathrm{mV}$ degree. The bio potential signal is the electrical signal of the $\mathrm{mV}$ degree produced by the body with the purpose to work regularly of the human body. Bio potential signals are the survival factors of living things such as blood pressure, heart rate, heart rhythm and brain functions. The ECG signal is obtained by taking the electrical signals generated by the heart with specific surface electrodes and generating an ECG signal to provide an idea about patient and diagnose.

In cable ECG devices, 6 chest probes and 3 peg type electrodes are generally used. The cost and deterioration of these electrodes is a major problem. It is misunderstood that ECG devices appear to be portable. The ECG device must be constantly on the patient's side. Another problem is that the electrodes must be removed in order to meet the basic needs of the patient. This leads to problems caused by the patient and the person interested in it. In patients with variable status and undergoing fibrillation, the electrodes of the ECG device should remain connected during the day.

When ECG device design is considered as a wireless, it will be improved in terms of cost, number of personnel and speed of intervention. One of the most important features is also that the ECG of more than one patient is transmitted to a single computer environment. Thus, the time and interest allocated to the patient will increase. In case of any problem will be taken information about patient and will be monitored continuously without affecting the daily activities of the patient.

ECG signals show the electrical activity of the heart. Patients can be monitored continuously while performing daily activities in household conditions. The sensors placed in the body of the patient are used to record the measurement data. The measured data is filtered and then displayed graphically using Matlab, Java, etc. software. This data is transmitted using wireless transmission to the doctor who examines the ECG signals and guides the patient if there is any emergency medical aid required and also monitors and keeps a track of all the patient's data periodically. Wireless transmission is made using the ZigBEE technique, which is an effective way to transfer data. The data from each sensor is detected using the USB link terminal on the computer. Then the data from a patient at home or hospital is transmitted to the doctor using ZigBEE.

This study aimed at establishing a portable and battery-operated meter read by a wireless connection with developing digital radio systems. 
In this study, analogue signals were measured with the help of the multi-channel ADC unit in Arduino processors, and this information was transferred to a USB extension device using the IEEE 802.15.4 (ZigBEE) radio network and from there it was transferred to PC for observation and storage. As a result, experimental data on the determination of the limits of the measurements made from the far distance have been obtained.

In this study, we present a realization of a ZigBEE based wireless ECG system for remotely monitoring the patient's electrocardiogram via wireless heart sensor (AD8232). The AD8232 offers a new alternative for measuring the heart rate remotely in real time during patient care. The presented wireless ECG system is composed of hardware such as Arduino microcontroller connected to the electrodes on the patient, personal laptop, tablet PC, smart phone etc. for ECG signal monitoring, AD8232 sensor for measuring heart rate, ZigBEE transceiver (XBee S2C) for wireless transmission of ECG signal, and software such as Serial Plot or Arduino Serial Plotter. The results from the tests show that the system designed over a single node can work seamlessly in an open area of approximately 20 to 100 meters and take ECG measurements wirelessly. Additionally, the obtained results from the tests show that the ECG measurements can be taken wirelessly within a range of 20 to 80 meters when the obstacles are also placed between ZigBEE transmitter and receiver. This range can be extended thanks to the ability of the ZigBEE to operate with nodes. This will provide the possibility for a patient to freely navigate in the room or in the hospital while the ECG measurements are being followed. Tests have shown that data can be obtained with high accuracy from the sensor units in motion.

The rest of the paper is organized as follows: Section 2 introduces the implementation of wireless ECG measurement system using ZigBEE (IEEE 802.15.4) standard. Section 2.1 explains the designed wireless ECG measurement system and measurement conditions. Section 3 presents the experimental studies to verify the feasibility and robustness of the designed wireless ECG measurement system and finally, the paper is concluded in Section 4.

\section{IMPLEMENTATION OF WIRELESS ECG MEASUREMENT SYSTEM USING ZigBEE (IEEE 802.15.4) STANDARD}

ZigBee transmitter / receiver (transceiver) units using the ISM 2400-2483 MHz band from radio frequency bands are manufactured as a single semiconductor chip. Therefore, this study should be evaluated primarily as the establishment of existing transmitter / receiver units and the investigation of implementation achievements. It is expected from this study that it is extended the use of wireless area networks by identifying the application features, problems and limits of the service belong to the developed technology.

The subject of the study is a communication system using IEEE 802.15.4 (ZigBEE) structure for use in a general purpose wireless measurement system. Experiments with the Arduino mini, capable of 200k sampling in the second, have shown that the measurement system can respond to the bandwidth requirement of approximately $20 \mathrm{kHz}$. Therefore, this shows that ZigBEE's communication limit of data rate of $250 \mathrm{kbits} / \mathrm{s}$ is reached. Real-time audio signals can be carried with such a measurement system. However, such systems that have low data rates and are not suitable for synchronous communication are not well suited for real-time audio and video transport applications. Since the IEEE 802.15.4 standard is a standard operating at very low power, the field units used can be fed with battery blocks. Thus, measurements made independent of the power grid can be much more sensitive.

Considering the characteristics of this general-purpose measuring system, biomedical signals can be carried by such a system. The combination of biomedical sensors and wireless sensor networks may have a very high potential in medical applications. Performance analyses of IEEE 802.15.4-based biomedical measurement systems have been found to be useful in evaluating transmission delay, end-to-end delay, and packet delivery rate in studies done (Liang \& Balasingham, 2007).

In this field, studies such as wearable ECG measurement system designs and smart home applications that can perform real-time health checks and seek help in risk situations have been done (Park et al., 2006), (Dağtaş et al., 2007).

This study aimed that realizing a general purpose sensor network with a wireless unit requiring high data rate or accurate measurement. 


\subsection{Designed Wireless ECG Measurement System}

The block diagram of the designed wireless ECG measurement system is shown in Figure 1.

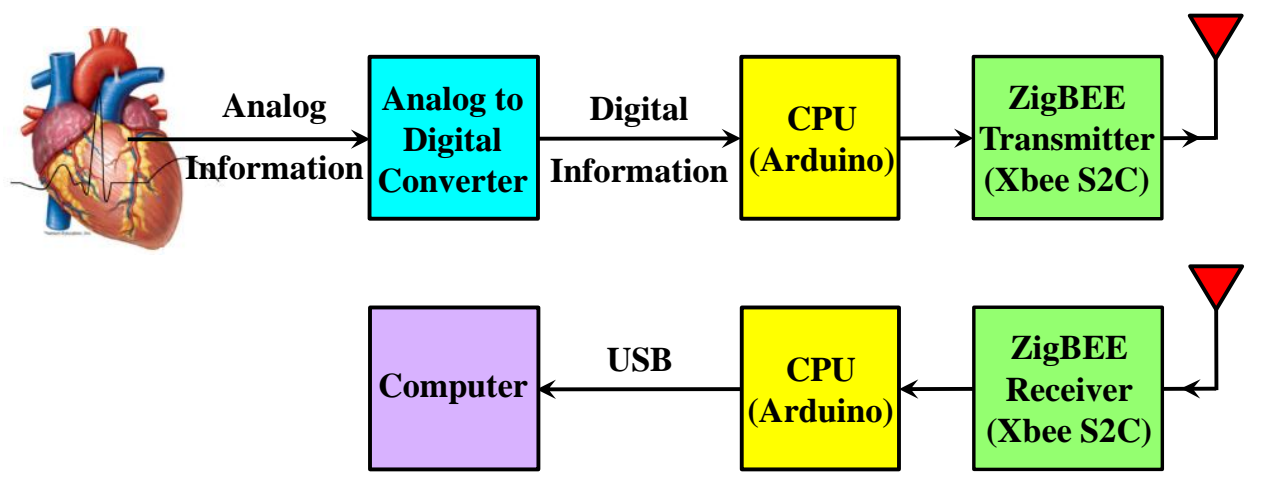

Figure 1: Block diagram of designed wireless ECG measurement system (Akbulut et al., 2017).

The analog signal from the heart sensor (AD8232) is digitally converted by a 10-bit analog to digital converter (ADC), the resulting digital information is processed by the Arduino mini-series processor, and with the Xbee S2C transmitter chip the radio frequency (RF) is also sent from the assigned channel. The signal on the RF channel is detected by the Xbee S2C series receiver chip on another sensor network unit in the receiver, demodulated and decoded, and is transmitted to the Arduino series processor. The Arduino processor transfers the information sequence it receives to the computer that it is connected via USB (universal serial bus). The program running on the computer draws the information sequence received via USB on the screen with the help of the Serial Plot or Arduino Serial Plotter program and displays the signal (Akbulut et al., 2017), (Margolis, 2011).

\section{EXPERIMENTAL STUDIES}

The experimental studies have composed of two stages. In the first stage studies are performed in open area with line of sight (LOS). In the second stage studies are implemented in none-LOS (N-LOS) environment when the obstacles are also placed between ZigBEE transmitter and receiver. The attenuation analysis of the obtained measurements in $\mathrm{dB}$ was made with the XCTU interface program (Akbulut et al., 2017).

\subsection{Experimental Results in LOS Environment}

In the first phase of the study, in order to test the operation of the wireless ECG measurement system measurements were taken when between the ZigBEE transmitter and the ZigBEE receiver units were in case of LOS in an open area.

Wireless ECG measurements were taken when the distance between the transmitter and receiver was 5 meters in Figure 2, 10 meters in Figure 4, 20 meters in Figure 6 and 30 meters in Figure 8. Additionally, in Figure 3, 5, 7 and 9, it is performed the attenuation analysis in $\mathrm{dB}$ for the same measurements.

Figure 2 shows the ECG chart when the distance between the transmitter and the receiver is 5 meters, and Figure 3 shows the $\mathrm{dB}$ attenuation analysis for this graph.

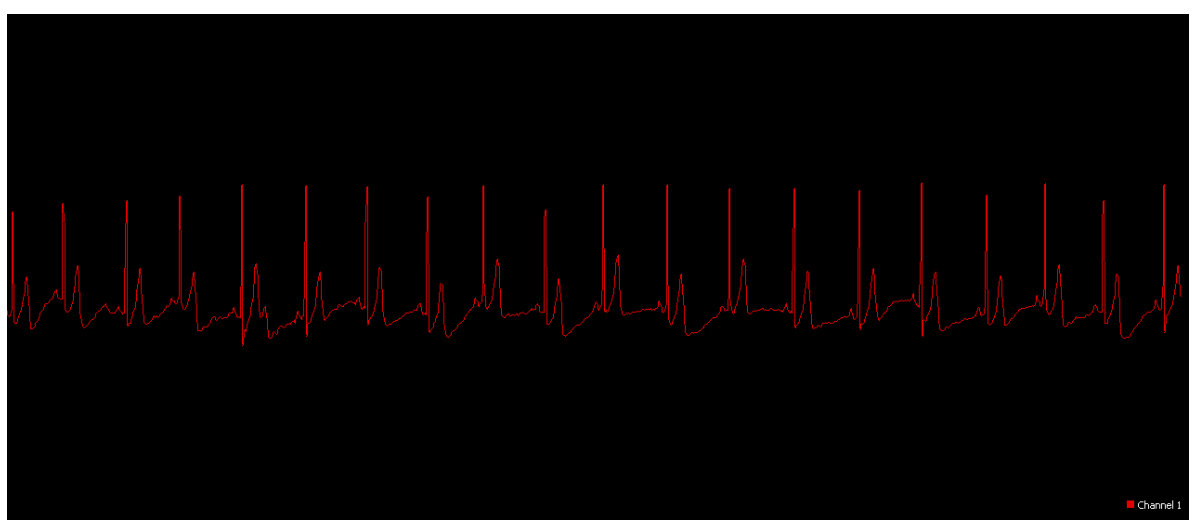

Figure 2: Wireless ECG measurement for distance of 5 meters. 
When Figure 2 is examined, it is seen that the ECG signal is obtained with high accuracy.

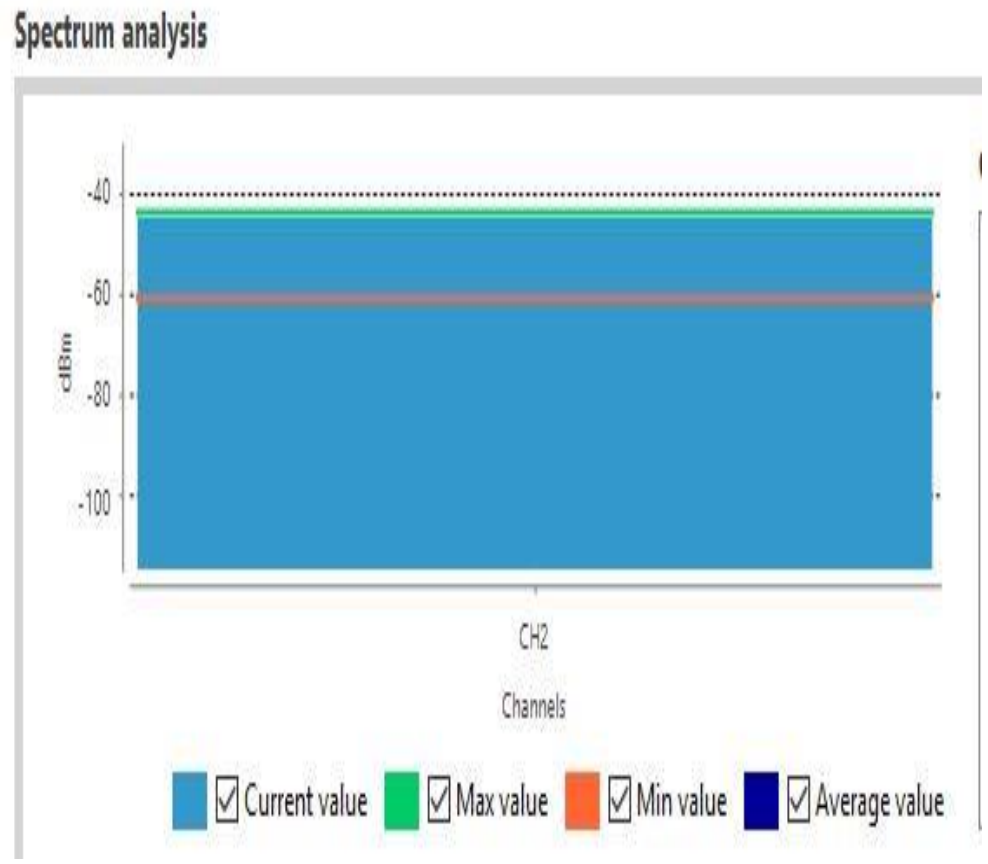

Figure 3: Attenuation analysis of wireless ECG measurement for distance of 5 meters.

When Figure 3 is investigated, it is determined that there is a loss of about $5 \mathrm{~dB}$ in the measured ECG signal. It is observed that Figures 2 and 3 confirm with each other.

Figure 4 shows the ECG graph measured at a distance of 10 meters between the transmitter and the receiver and the dB attenuation analysis of this graph is shown in Figure 5.

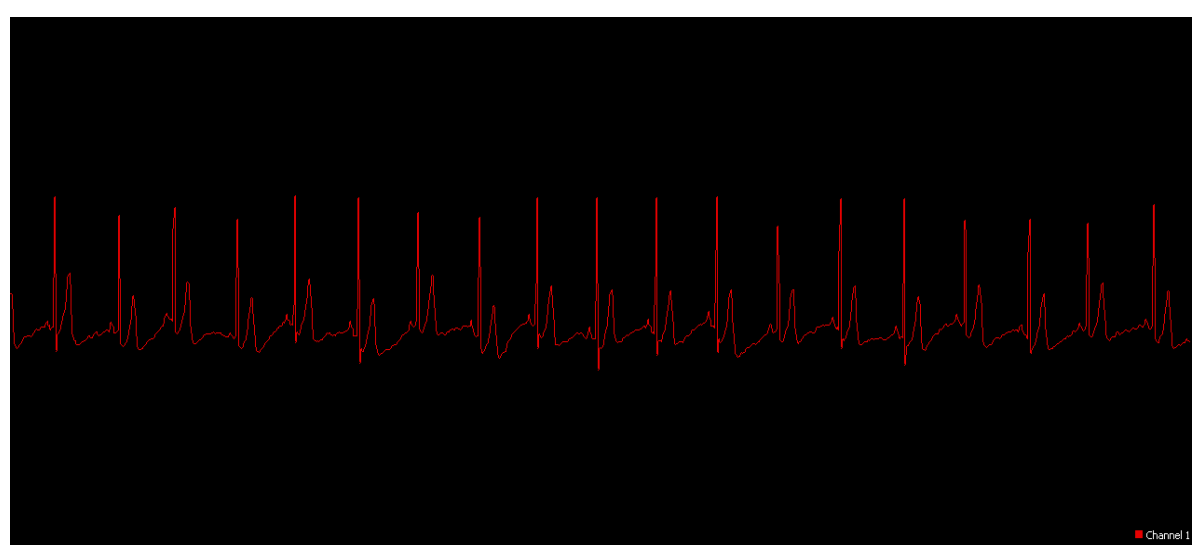

Figure 4: Wireless ECG measurement for distance of 10 meters.

When Figure 4 is examined, it is seen that the ECG signal is similarly obtained with high accuracy. 


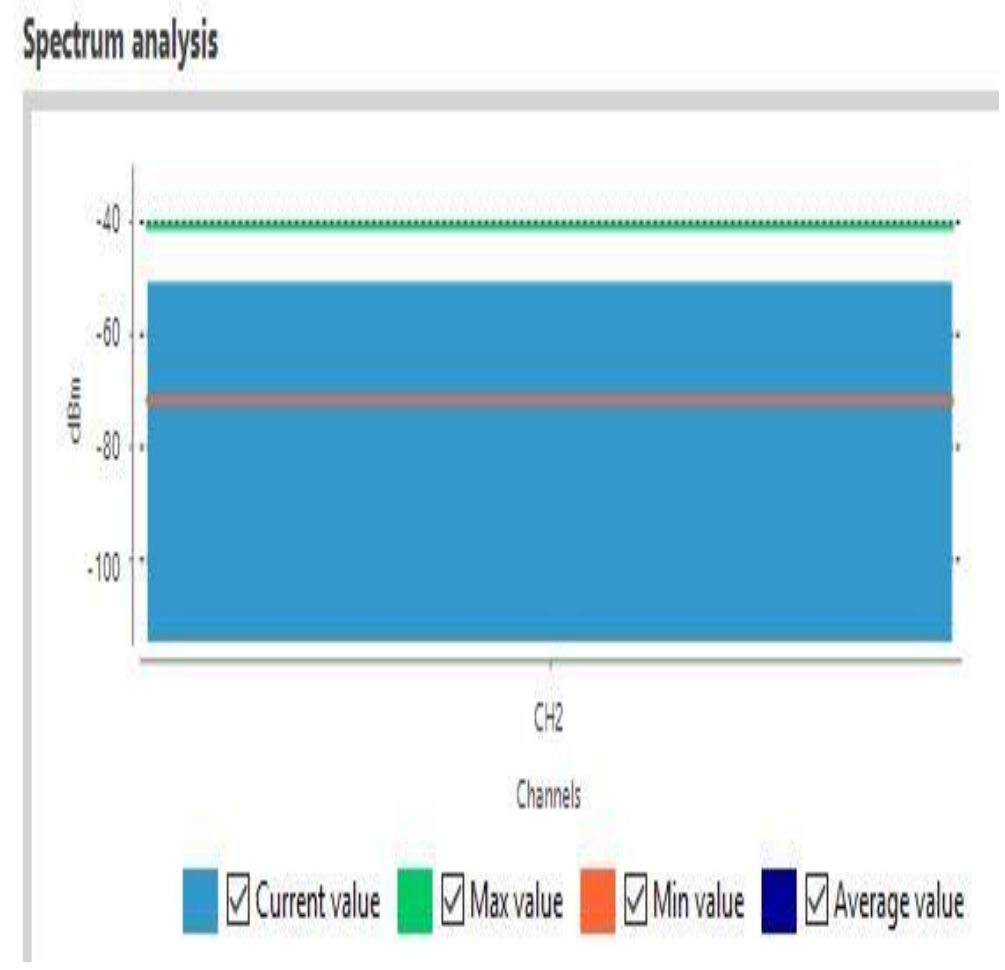

Figure 5: Attenuation analysis of wireless ECG measurement for distance of 10 meters.

When Figure 5 is investigated, it is determined that there is a loss of about $10 \mathrm{~dB}$ in the measured ECG signal. It is observed that Figures 4 and 5 verify with each other.

Figure 6 shows the ECG graph measured at a distance of 20 meters between the transmitter and the receiver, and the $\mathrm{dB}$ attenuation analysis of this graph is shown in Figure 7.

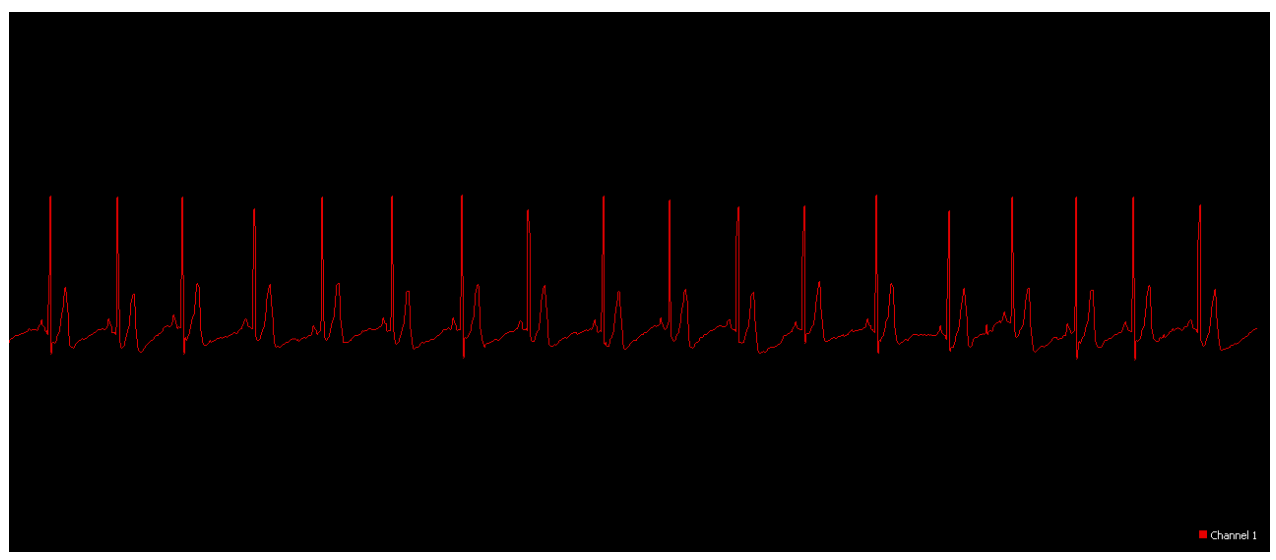

Figure 6: Wireless ECG measurement for distance of 20 meters.

When Figure 6 is investigated, it is seen that impairments in the ECG signal are increased. 


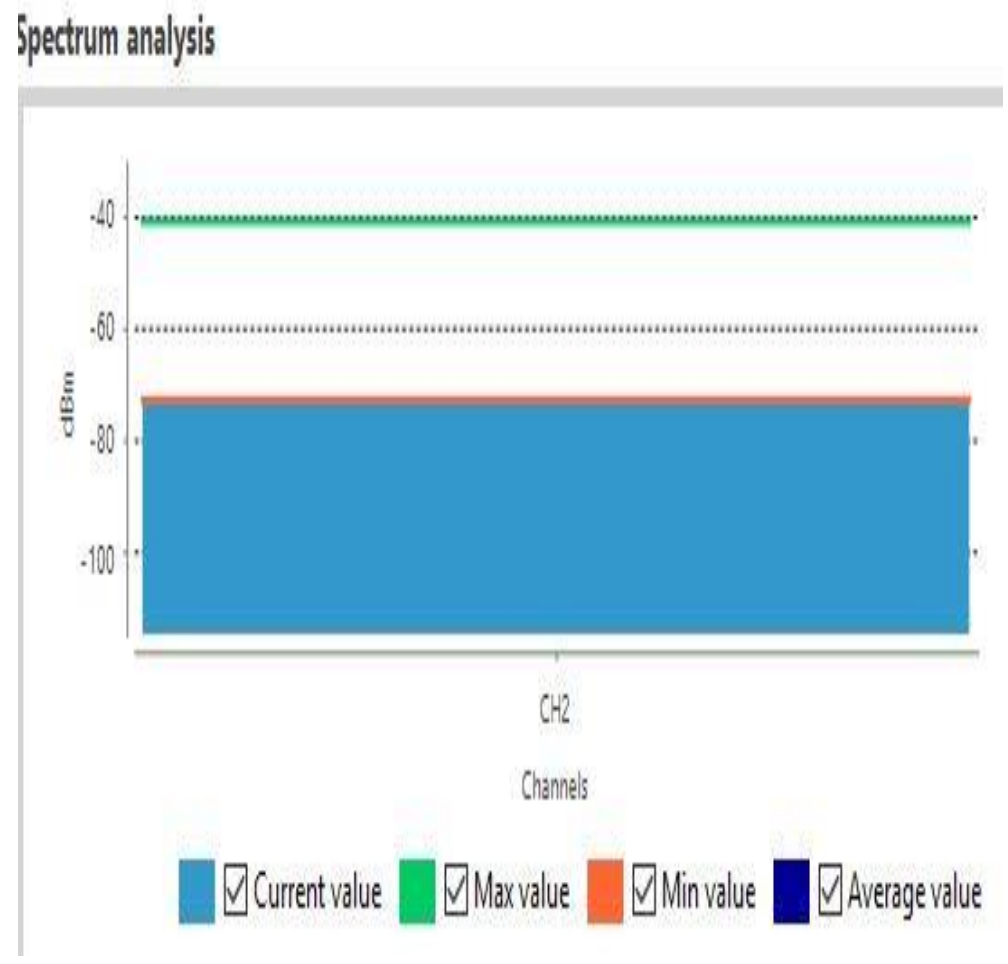

Figure 7: Attenuation analysis of wireless ECG measurement for distance of 20 meters.

When Figure 7 is examined, it is determined that there is a loss of about $35 \mathrm{~dB}$ in the measured ECG signal.

Figure 8 shows the measured ECG graph when the distance between the transmitter and the receiver is 30 meters and Figure 9 shows the $\mathrm{dB}$ attenuation analysis of this graph.

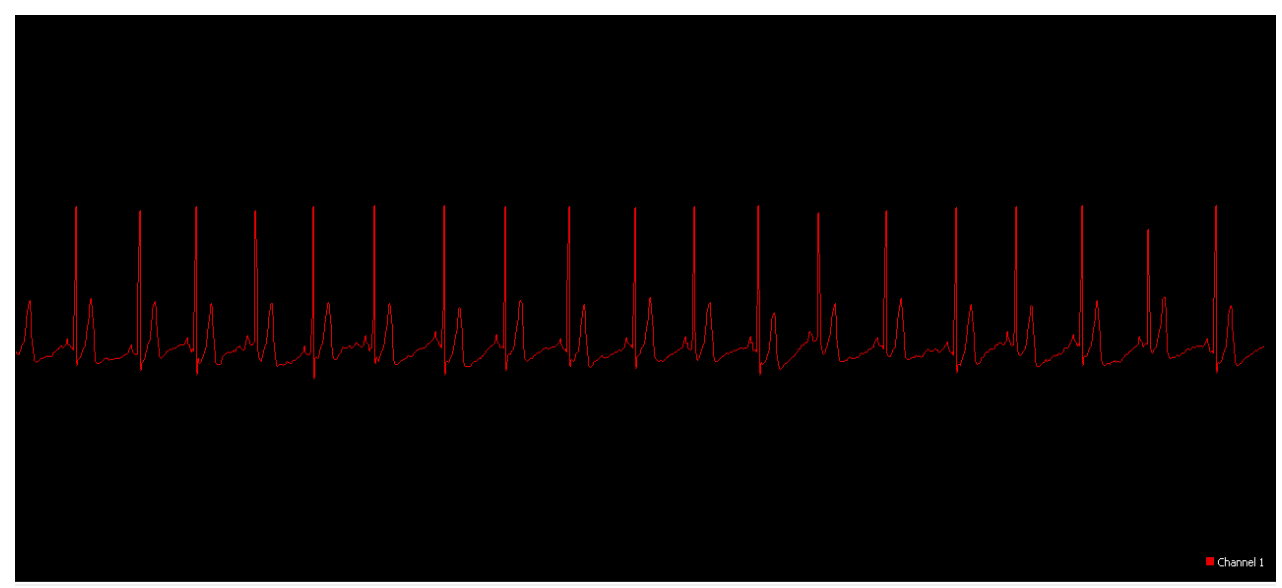

Figure 8: Wireless ECG measurement for distance of 30 meters.

When Figure 8 is investigated, it is seen that the deteriorations in the ECG signal increases more. Figure 9 shows that there is a loss of approximately $55 \mathrm{~dB}$ in the measured ECG signal. 


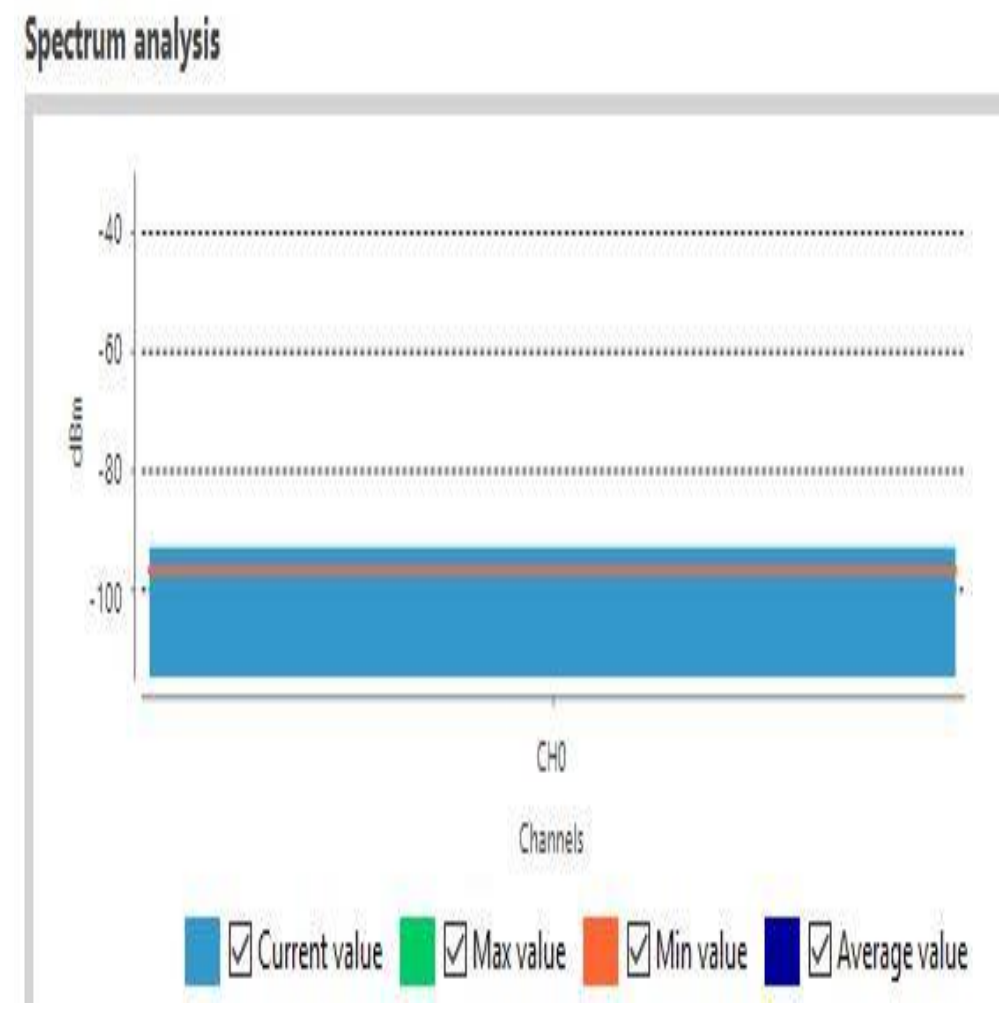

Figure 9: Attenuation analysis of wireless ECG measurement for distance of 30 meters.

\subsection{Experimental Results in N-LOS Environment}

In the second phase of the study, in order to test the operation of the wireless ECG measurement system measurements were taken where the ZigBEE transmitter and the ZigBEE receiver units did not see each other (in case of N-LOS).

In Figure 10, wireless ECG measurements are taken in case of the distance between the transmitter and the receiver is 10 meters and the door obstruction. Also, in Figure 11, the attenuation analysis is performed in terms of dB of this measurement.

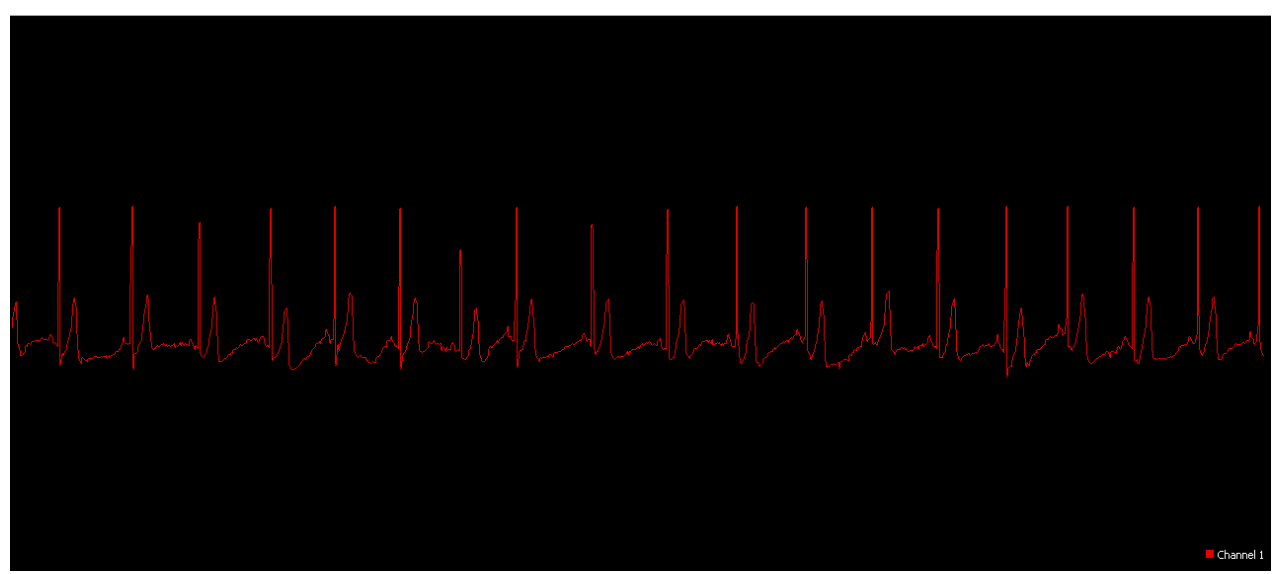

Figure 10: Wireless ECG measurement when there is door obstacle for distance of 10 meters.

When Figure 10 is investigated, it is seen that the ECG signal is distorted due to the door obstruction. When Figure 11 is examined, it is determined that there is a loss of about $20 \mathrm{~dB}$ in the measured ECG signal. 


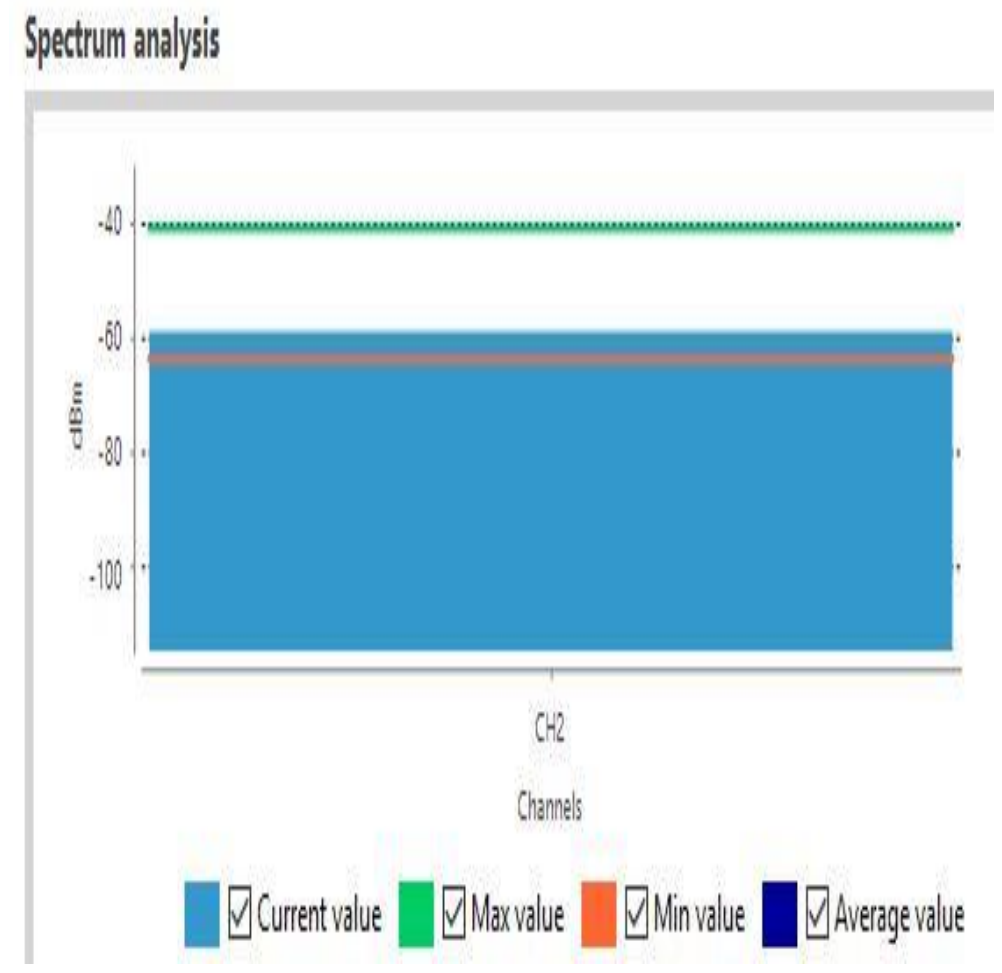

Figure 11: Attenuation analysis of wireless ECG measurement when there is door obstacle for distance of 10 meters.

In Figure 12, wireless ECG measurements were taken in case of the transmitter is on the ground floor and the receiver is on the upper floor (first floor).

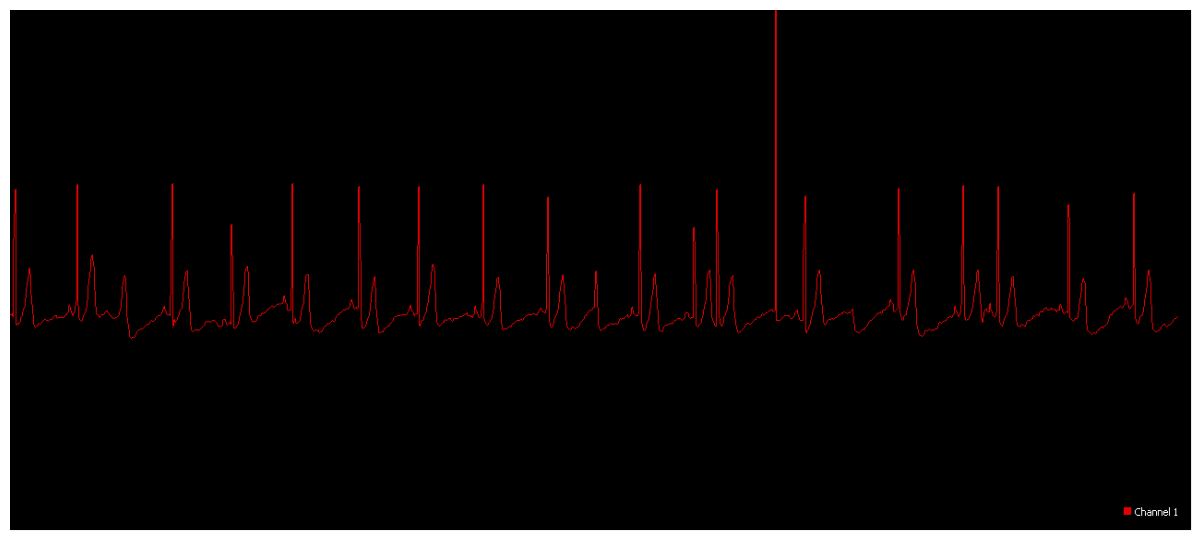

Figure 12: Wireless ECG measurement when the transmitter is on the ground floor and the receiver is on the first floor.

When Figure 12 is investigated, it is seen that there are corruptions in the ECG signal due to the layer between the transmitter and the receiver.

In Figure 13, wireless ECG measurements were taken when the transmitter is on the ground floor and the receiver is on the two upper floor (second floor). 


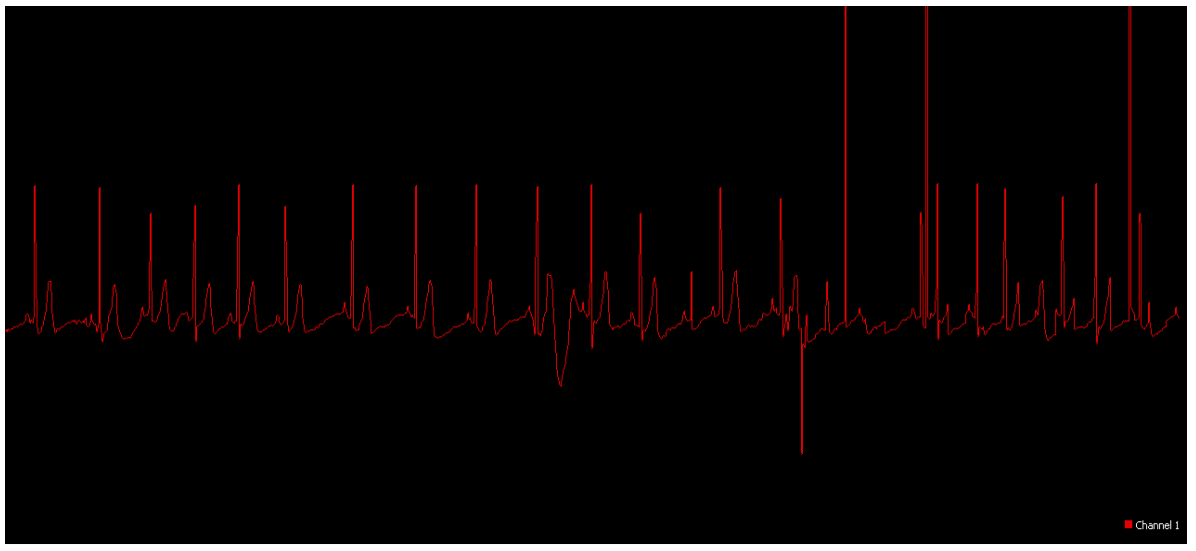

Figure 13: Wireless ECG measurement when the transmitter is on the ground floor and the receiver is on the second floor.

When Figure 13 is examined, it is seen that the deterioration increases in the ECG signal due to the fact that there are two layers between the transmitter and the receiver.

In Figure 14, wireless ECG measurements were taken when the transmitter is on the ground floor and the receiver is on the upper three layers (third floor).

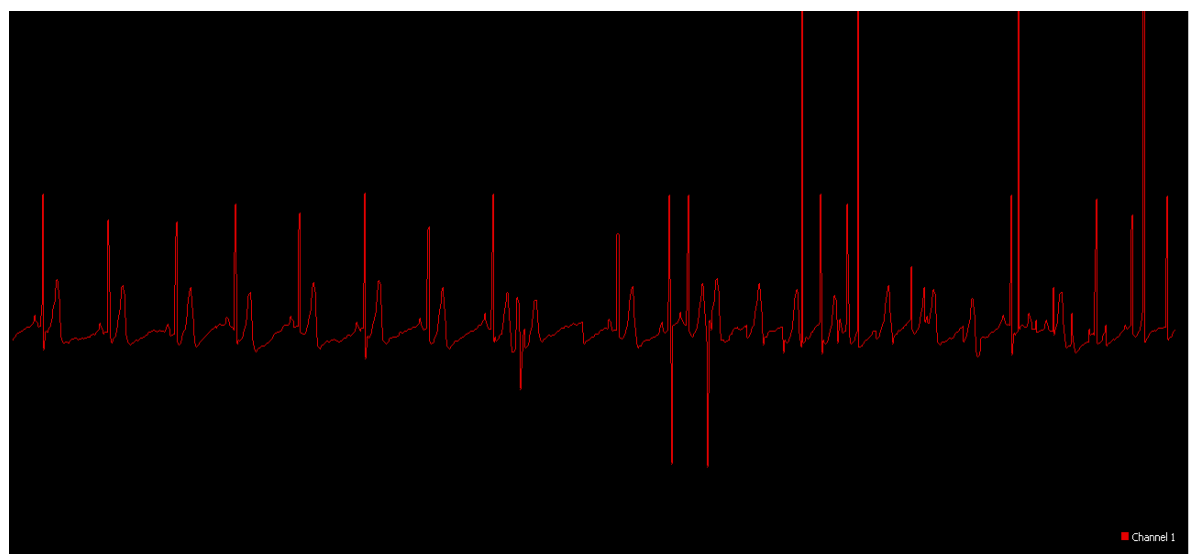

Figure 14: Wireless ECG measurement when the transmitter is on the ground floor and the receiver is on the third floor.

When Figure 14 is investigated, it is observed that the deterioration of the ECG signal has increased to an extreme extent due to the three floors between the transmitter and the receiver.

In Figure 15, measurements of the designed wireless ECG device have been compared simultaneously with measurements of real ECG device in the hospital environment. In a real ECG device, 6 electrodes are attached to the chest and 4 electrodes are attached to the hands and ankles. This comparison of these ECG signals shows what extent the ECG signal is taken correctly from the body and transmitted wirelessly.

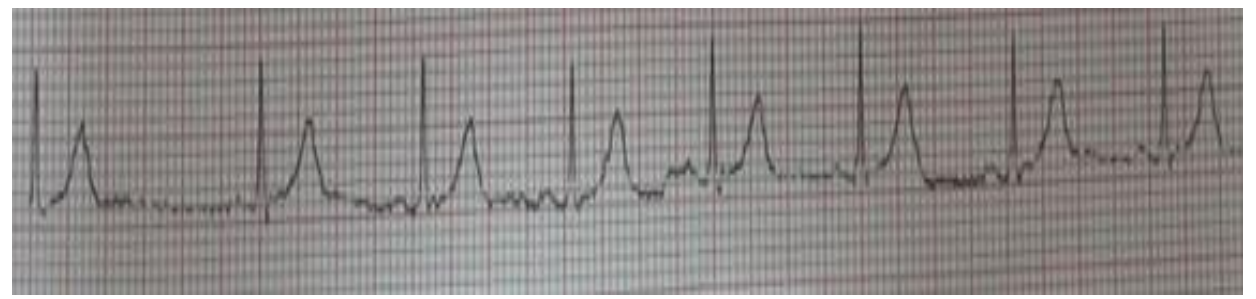

a) Real ECG measurement 


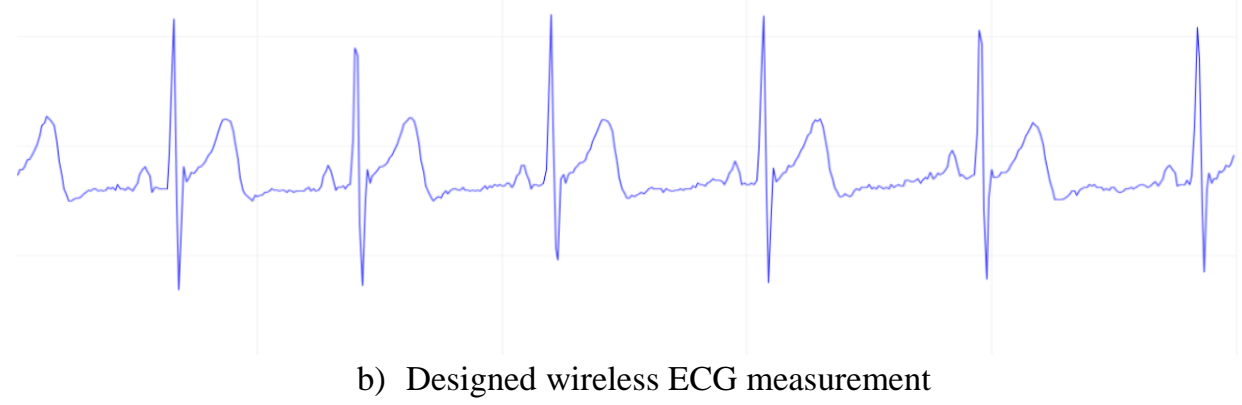

Figure 15: Comparison of the designed wireless ECG measurements with real wired ECG.

When Figure 15 is examined, it is seen that the measurements taken with the designed wireless ECG system are approximately the same as the real ECG measurement. As a result, the ECG signal taken from any person is transmitted wirelessly to the intended system without any problems.

\section{CONCLUSIONS}

Wearable and mobile systems continue to evolve rapidly in order to save the patient from disturbing cable extensions and be able to follow the required measurements on the move. In this context, the structure of the wireless sensor networks and the installation methods were investigated and the implementation of a wireless ECG measurement system using IEEE 802.15.4 (ZigBEE) was performed in this study.

The results from the tests show that the system designed over a single node can work seamlessly in an open area of approximately 20 to 100 meters and take ECG measurements wirelessly. In addition, the results from the tests show that the ECG measurements can be taken wirelessly within the range of 20 to 80 meters when there are obstacles between the ZigBee transmitter and the receiver.

\section{REFERENCES}

Akbulut A., Ekin Ş., Tunç Ö. (2017), Implementation of ZigBEE (IEEE 802.15.4) Based Wireless ECG System, Bachelor Thesis, Nuh Naci Yazgan University, Department of Electrical and Electronics Engineering, Kayseri, Turkey.

Dağtaş S., Pekhteryev G. and Şahinoğlu Z. (2007), "Multi-stage Real Time Health Monitoring via ZigBEE in Smart Homes".

IEEE Standard (2007), IEEE Standard for Part 15.4: Wireless Medium Access Control (MAC) and Physical Layer (PHY) specifications for Low Rate Wireless Personal Area Networks (LR-WPANs).

Liang X. and Balasingham I. (2007), "Performance Analysis of The IEEE 802.15.4 Based ECG Monitoring Network".

Margolis M. (2011), “Arduino Cookbook”, First Edition, Chapter 14.

Park C., Chou P., Bai Y., Matthews R. and Hibbs A. (2006), “An Ultra Wearable, Wireless, Low Power ECG Monitoring System”.

Romer K. and Friedemann M. (2004), “The Design Space of Wireless Sensor Networks”, 54-61.

Somay A. (2009), "Implementation of A Wireless Measurement Probe using IEEE 802.15.4 (ZigBEE) Standard", Ms.C. Thesis, Graduate School of Natural and Applied Science, Black Sea Technical University, Trabzon. 